Review

\title{
The genus Inula and their metabolites: From ethnopharmacological to medicinal uses
}

\author{
Ana M.L. Seca ${ }^{\mathrm{a}, \mathrm{b}}$, Alice Grigore ${ }^{\mathrm{c}}$, Diana C.G.A. Pinto ${ }^{\mathrm{b}}$, Artur M.S. Silva ${ }^{\mathrm{b}, *}$ \\ a DCTD, University of Azores, 9501-801 Ponta Delgada, Portugal \\ ${ }^{\mathrm{b}}$ Chemistry Department \& QOPNA, University of Aveiro, Campus de Santiago, 3810-193 Aveiro, Portugal \\ ${ }^{\mathrm{c}}$ Department of Pharmaceutical Biotechnologies, National Institute of Chemical-Pharmaceutical R\&D, 112 Vitan Av., Bucharest, Romania
}

\section{A R T I C L E I N F O}

\section{Article history:}

Received 27 October 2013

Received in revised form

3 April 2014

Accepted 5 April 2014

Available online 19 April 2014

\section{Keywords:}

Inula

Traditional medicinal plants

Sesquiterpene lactones

Cytotoxicity

Anti-inflammatory

Antimicrobial

\begin{abstract}
A B S T R A C T
Ethnopharmacological relevance: The genus Inula comprises more than one hundred species widespread in temperate regions of Europe and Asia. Uses of this genus as herbal medicines have been first recorded by the Greek and Roman ancient physicians. In the Chinese Pharmacopoeia, from the 20 Inula spp. distributed in China, three are used as Traditional Chinese medicines, named Tumuxiang, Xuanfuhua and Jinfeicao. These medicines are used as expectorants, antitussives, diaphoretics, antiemetics, and bactericides. Moreover, Inula helenium L. which is mentioned in Minoan, Mycenaean, Egyptian/Assyrian pharmacotherapy and Chilandar Medical Codex, is good to treat neoplasm, wound, freckles and dandruff. Many other Inula spp. are used in Ayurvedic and Tibetan traditional medicinal systems for the treatment of diseases such as bronchitis, diabetes, fever, hypertension and several types of inflammation. This review is a critical evaluation of the published data on the more relevant ethnopharmacological and medicinal uses of Inula spp. and on their metabolites biological activities. This study allows the identification of the ethnopharmacological knowledge of this genus and will provide insight into the emerging pharmacological applications of Inula spp. facilitating the prioritirization of future investigations. The corroboration of the ethnopharmacological applications described in the literature with proved biological activities of Inula spp. secondary metabolites will also be explored.
\end{abstract}

Abbreviations: A-549, human lung carcinoma cell line; ABTS, 2,2'-azinobis(3-ethylbenzothiazoline-6-sulfonic acid); AGS, human gastric carcinoma cell line; AHR, airway hiper-responsiveness; AKT, serine/threonine-specific protein kinase; AMPK, adenosine 5'-monophosphate-activated protein kinase; ATPase, adenosine triphosphatase; B16, murine melanoma cell line; $\mathrm{Bcl}-2$, apoptosis regulator proteins encoded by the BCL2 gene; Capan-2, human pancreas adenocarcinoma cell line; $\mathrm{CC}_{50}$, $50 \%$ cytotoxic concentration; c-FLIP, cellular FLICE (FADD-like interleukine-1 $\beta$-converting enzyme) inhibitory protein; COX, cyclooxygenase; COX-1, cyclooxygenase-1; COX-2,

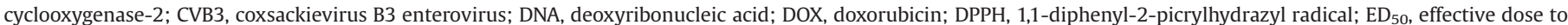
produce effect in $50 \%$ of a population; G1, astrocytoma cell line; G2/M, cell cycle checkpoint in eukaryotic organisms; GMK, green monkey kidney cell line; GSTs, glutathioneS-transferase; HCT-116, colon carcinoma cell line; HeLa, human cervix cancer cell line; HepG-2, liver hepatocellular carcinoma cell line; HIV/AIDS, human immunodeficiency virus infection/acquired immunodeficiency syndrome; HIV-1, human immunodeficiency virus type 1; HL-60, human promyelocytic leukemia cell line; HSP70, heat shock protein 70; HSV-1, herpes Simplex Virus type 1; HT-29, human colon adenocarcinoma grade II cell line; IC 50 , inhibitory concentration for $50 \%$ of viability; ICAM-1, intracellular adhesion molecule-1; IKK, IкB kinase; IKK $\beta$, IкB kinase $\beta$; IL-2, Interleukin 2; iNOS, inducible nitric oxide synthase; JNK, c-jun terminal-NH2 kinase; KB, human nasopharyngeal carcinoma; L6, rat skeletal myoblasts cell line; L929, murine aneuploid fibrosarcoma cell line; L929sA, murine fibrosarcoma cell line; L-NAME, N $\omega$-nitro-Larginine methyl ester; LOVO, human colon adenocarcinoma cell line; LPS, lipopolysaccharide; LTB4, leukotriene B4; MBC, minimum bactericidal concentrations; MCF-7, breast cancer cell line; MDA-MB-435, human breast adenocarcinoma cells; MDCK, madin-Darby canine kidney cell line; MGC-803, human gastric carcinoma cell line; MIC, minimum inhibitory concentrations; MMP-9, matrix metalloproteinase-9; MRSA, methicillin resistant Staphylococcus aureus; MSSA, methicillin-sensitive Staphylococcus aureus; NCI, national Cancer Institute; NF-кB, nuclear factor kappa B; Nrf2, nuclear factor 2-related factor 2; OPN, osteopontin; p47phox, 47-kilodalton cytosolic subunit of the multi-protein complex known as NADPH oxidase; PAF, platelet-activating factor; PBMCs, human peripheral blood mononuclear cells; PC-3, human prostate cancer cell lines; PDGF, platelet-derived growth factor; PGE2, prostaglandin E2; PI3K, phosphatidylinositol 3-kinase; PLA2, phospholipase A2; PMA, phorbol myristate acetate; PMNs, polymorphonuclear leukocytes; PPAR $\gamma 2$, peroxisome proliferator-activated receptor $\gamma 2$; QR, quinone reductase; RAW264, murine monocyte/macrophage line derived from ascitic tumour induced with Abelson leukaemai virus; ROS, reactive oxygen species; SCF, stem cell factor; SGC-7901, gastric cancer cell line; SiHa, human cervix uteri cancer cell line; SK-28, human melanoma cell line; SOD, superoxide dismutase; sPLA2, secretory phospholipase A2; STAT1, signal transducer and activator of transcription 1; SW620, human colon carcinoma cell line; Th2, T helper cells type 2; TNFR1, tumour necrosis factor receptor 1; TNF- $\alpha$, tumour necrosis factor-alpha; TPA, 12-Otetradecanoylphorbol-13-acetate; Tyrp1, tyrosinase-related protein 1; U937, human monoblastic leukemia cell line; VCAM-1, vascular adhesion molecule-1; Vero, African green monkey kidney cell line; VSMCs, vascular smooth muscle cells

* Corresponding author. Tel.: +351234370714; fax: +351234370084

E-mail addresses: anaseca@uac.pt (A.M.L. Seca), alicearmatu@yahoo.com (A. Grigore), diana@ua.pt (D.C.G.A. Pinto), artur.silva@ua.pt (A.M.S. Silva). 
Materials and methods: The major scientific databases including ScienceDirect, Medline, Scopus and Web of Science were queried for information on the genus Inula using various keyword combinations, more than 180 papers and patents related to the genus Inula were consulted. The International Plant Name Index was also used to confirm the species names.

Results: Although the benefits of Inula spp. are known for centuries, there are insufficient scientific studies to certify it. Most of the patents are registered by Chinese researchers, proving the traditional use of these plants in their country. Although a total of sixteen Inula species were reported in the literature to have ethnopharmacological applications, the species Inula cappa (Buch.-Ham. ex D.Don) DC., Inula racemosa Hook. f., Inula viscosa (L.) Aiton [actually the accepted name is Dittrichia viscosa (L.) Greuter], Inula helenium, Inula britannica L. and Inula japonica Thunb. are the most frequently cited ones since their ethnopharmacological applications are vast. They are used to treat a large spectrum of disorders, mainly respiratory, digestive, inflammatory, dermatological, cancer and microbial diseases. Fifteen Inula spp. crude extracts were investigated and showed interesting biological activities. From these, only 7 involved extracts of the reported spp. used in traditional medicine and 6 of these were studied to isolate the bioactive compounds. Furthermore, 90 bioactive compounds were isolated from 16 Inula spp. The characteristic compounds of the genus, sesquiterpene lactones, are involved in a network of biological effects, and in consequence, the majority of the experimental studies are focused on these products, especially on their cytotoxic and anti-inflammatory activities. The review shows the chemical composition of the genus Inula and presents the pharmacological effects proved by in vitro and in vivo experiments, namely the cytotoxic, anti-inflammatory (with focus on nitric oxide, arachidonic acid and NF-кB pathways), antimicrobial, antidiabetic and insecticidal activities.

Conclusions: Although there are ca. 100 species in the genus Inula, only a few species have been investigated so far. Eight of the sixteen Inula spp. with ethnopharmacological application had been subjected to biological evaluations and/or phytochemical studies. Despite Inula royleana DC. and Inula obtusifolia A. Kerner are being used in traditional medicine, as far as we are aware, these species were not subjected to phytochemical or pharmacological studies. The biological activities exhibited by the compounds isolated from Inula spp., mainly anti-inflammatory and cytotoxic, support some of the described ethnopharmacological applications. Sesquiterpene lactone derivatives were identified as the most studied class, being britannilactone derivatives the most active ones and present high potential as anti-inflammatory drugs, although, their pharmacological effects, dose-response relationship and toxicological investigations to assess potential for acute or chronic adverse effects should be further investigated. The experimental results are promising, but the precise mechanism of action, the compound or extract toxicity, and the dose to be administrated for an optimal effect need to be investigated. Also human trials (some preclinical studies proved to be remarkable) should be further investigated. The genus Inula comprises species useful not only in medicine but also in other domains which makes it a high value-added plant.

(c) 2014 Elsevier Ireland Ltd. All rights reserved.

\section{Contents}

1. Introduction. . . . . . .

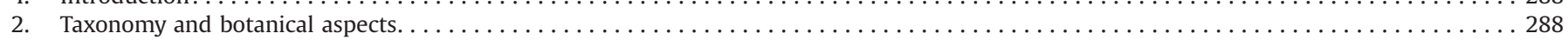

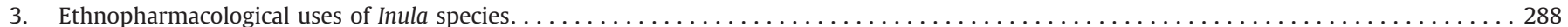

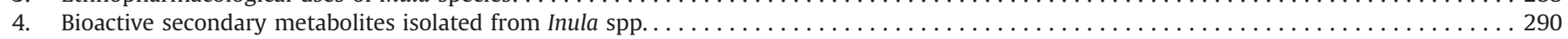

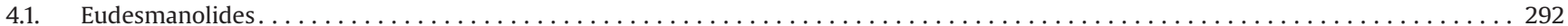

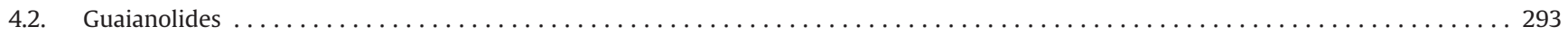

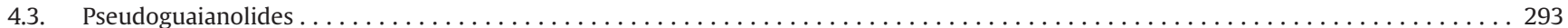

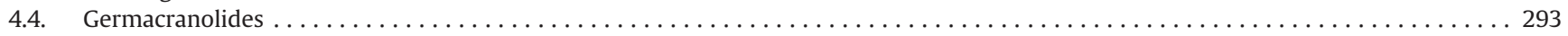

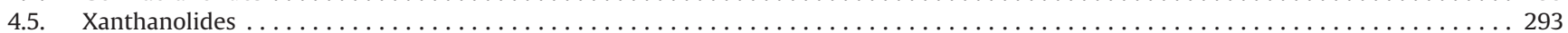

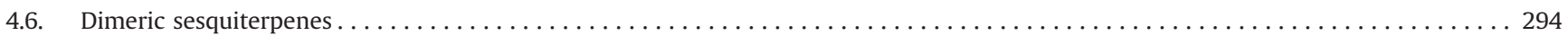

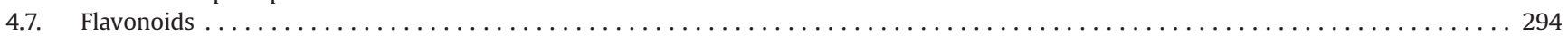

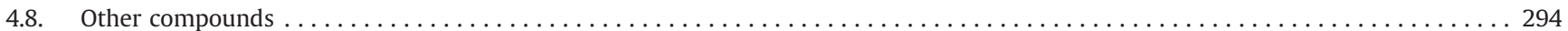

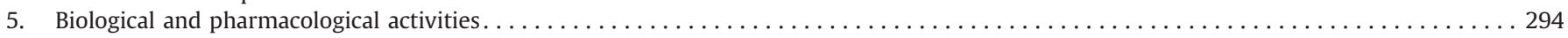

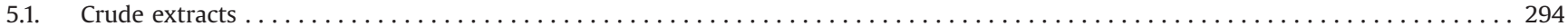

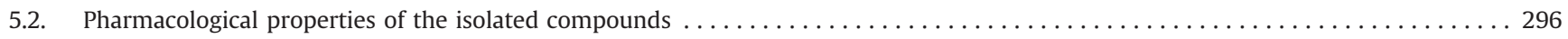

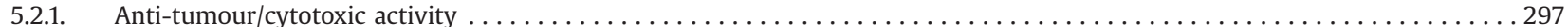

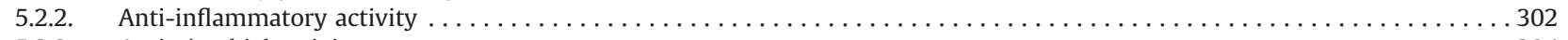

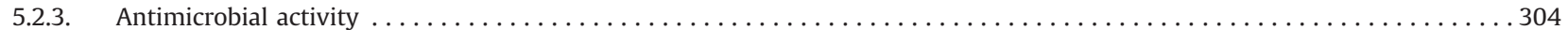

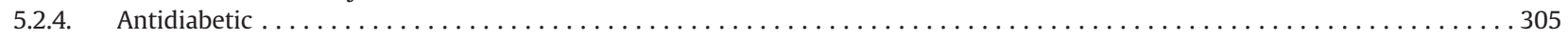

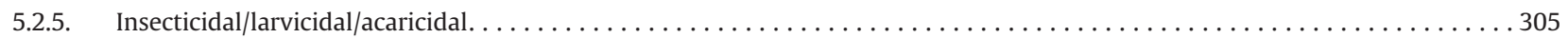

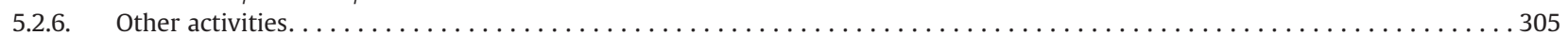

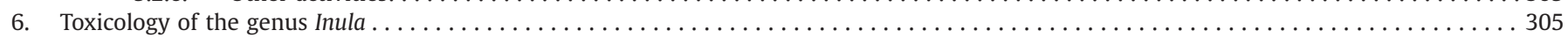

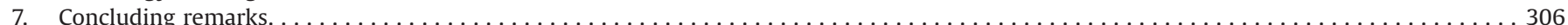

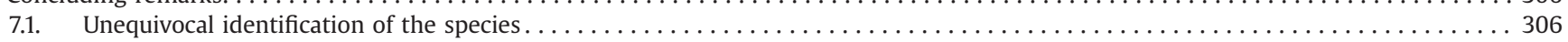

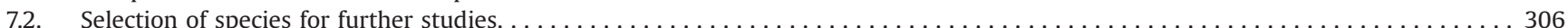

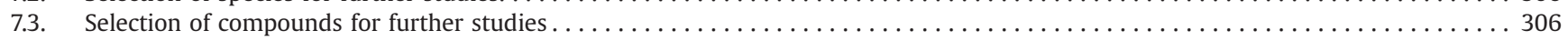

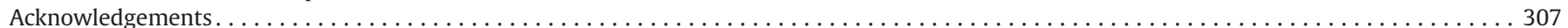

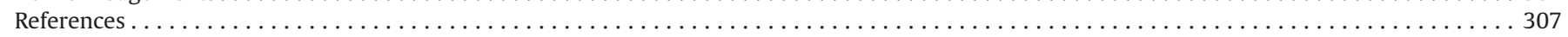

